\title{
KLEINIAN GROUPS, LAPLACIAN ON FORMS AND CURRENTS AT INFINITY
}

\author{
MARK POLLICOTT \\ (Communicated by Clifford J. Earle, Jr.)
}

\begin{abstract}
In this note we consider the spectrum of the Laplacian acting on the space of (co-closed) differential forms on the quotient of $n$-dimensional hyperbolic space by a co-compact Kleinian group. Using a result of P.-Y. Gaillard we relate these to currents on the sphere at infinity of hyperbolic space with distinctive transformation properties under the action of the group. We analyse these currents using zeta-functions and Ruelle's Transfer operator. This represents a partial extension of earlier work of the author related to Fuchsian groups. In an appendix we propose an alternative approach to related questions.
\end{abstract}

\section{INTRODUCTION}

The purpose of this note is to clarify the interaction of the objects in the title and to investigate their relationship with the "Transfer" operators (in the sense of Ruelle) which play an important role in symbolic dynamics and ergodic theory.

We shall chiefly be concerned with co-compact Kleinian groups $\Gamma$ (which are discrete groups of isometries of $n$-dimensional hyperbolic space). Work of Patterson [11] and Sullivan [15] has helped to understand the relationship between the first eigenvalue of the Laplacian on functions for $V=H^{n} / \Gamma$ and a distinguished measure $m$ on the sphere at infinity $S^{n-1}$ (supported on the limit set $\Lambda$ ). In [13] we observed that, for Fuchsian groups, the other eigenvalues of the Laplacian could be understood in terms of certain distributions on the sphere at infinity (via a result of Helgason [10]), and then in terms of transfer operators. In this article we shall show that, after some necessary modifications, this correspondence continues for eigenvalues of the Laplacian on (co-closed) forms and currents on the sphere at infinity (i.e., linear functionals on forms). In particular, these currents can be approached in terms of transfer operators on forms at infinity. We shall also consider the relationship with zeta functions.

Besides purely aesthetic appeal, this analysis has the advantage of presenting an approach to Laplacian spectra which seems particularly amenable to explicit

Received by the editors March 17, 1989.

1980 Mathematics Subject Classification (1985 Revision). Primary 58F19; 30F40.

Key words and phrases. Kleinian groups; Laplacian; differential forms; transfer operator; zeta function; limit set. 
computation. The classical approaches to estimating these spectra are usually somewhat less successful for forms than for functions. However, the characterisation in terms of transfer operators does not appear to be appreciably different.

In the appendix we take the opportunity to discuss different approaches to obtain estimates for eigenvalues of the Laplacian.

\section{LAPLACIAN AND CURRENTS}

Let $H^{n}$ denote $n$-dimensional hyperbolic space, with the usual Poincare metric, and let $S^{n-1}$ denote the sphere at infinity, which is the unit sphere in the disc model for hyperbolic space. Let $V=H^{n} / \Gamma$, where $\Gamma \subseteq \operatorname{Isom}\left(H^{n}\right)$ is a discrete group, be a compact manifold.

For $0 \leq p \leq n$ let $E_{p}$ denote the $p$-forms on $V$. We shall use the standard notation for the familiar linear operators $d: E_{p} \rightarrow E_{p+1}, *: E_{p} \rightarrow E_{n-p}$, and $\delta: E_{p} \rightarrow E_{p-1}$ (a nice account is given in chapter 6 of [17]). The Laplacian on $p$-forms is defined by $p=d \delta+\delta d$ (for $p=0, E_{0}$ reduces to functions and $\Delta_{0}$ becomes the familiar differential operator). A $p$-form $w$ is called closed if $d w=0$; co-closed if $\delta w=0$ and harmonic if $\Delta_{p} w=0$ (for the special case $p=0$, i.e., functions, forms are trivially co-closed). Recall the simple fact that a $p$-form is harmonic if and only if it is both closed and co-closed, c.f. [6].

We can lift the Laplacian $\Delta_{p}$ on $p$-forms for $V$ to a Laplacian $\Delta_{p}$ for $p$-forms for $H^{n}$. In particular, there is a bijection between eigenforms $w$ for $\Delta_{p}$ and eigenforms $w$ for $\Delta_{p}$ with the additional property that they are automorphic under $\Gamma$ (i.e., $w(x)=w(g x), g(\Gamma))$.

For $p=0$ Helgason describes a correspondence between eigenfunctions for $\Delta_{0}$ and (analytic) distributions on $S^{n-1}$. For $0 \leq p<n$, Gaillard has given a similar characterisation of (co-closed) eigenforms for $\Delta_{p}$ in terms of (analytic hyper-) distributions on $S^{n}$. This latter result leads to the following kind of result.

A $p$-current on $S^{n-1}$ is a linear functional on $p$-forms on $S^{n-1}$.

Theorem 1. There is a bijection between:

(a) co-closed eigenforms $\Delta_{p} w=-\lambda w$ on $V$; and

(b) p-currents on $S^{n}$ which satisfy the condition

$$
g^{*} v=\|D g\|^{s} v, \quad \text { where } 0 \leq p<n-1,
$$

where $s=[n-1] / 2+i \beta$ when $\lambda=[n-1]^{2} / 4+\beta^{2}$, providing $s$ is not one of the exceptional values $s=n, n+1, n+2, \ldots$, or $[n-1] / 2$.

(NB. Here we have used the notation $\left.\left\|D_{x} g\right\|=\left|\operatorname{det}\left(D_{x} g\right)\right|^{1 / n}\right)$.

Proof. This is a straightforward deduction from Gaillard's nice work, c.f. [7]. He gives a characterisation of co-closed forms on $H^{n}$ in terms of (analytic hyper-) distributions on $S^{n-1}$, c.f. [7, pp. 591-2]. In addition, by virtue of their constrained growth (they are in fact bounded), they actually correspond to 
smooth (i.e., Schwartzian) currents on $S^{n-1}$ (this also appears in [7, p. 592]). In view of the automorphic property of this characterisation, the work of Gaillard shows that the corresponding currents transform as described by (1) (c.f. [13] for the special case $p=0, n=2$, based on Helgason's result). The details of this more general case are similar.

Corollary 1.1. There is a bijection between:

(a) harmonic p-forms on $V$,

(b) p-currents on $S^{n-1}$ which satisfy the condition

$$
g^{*} v=\|D g\|^{[n-1]} v \text {. }
$$

The Laplacian is known to extend to a self-adjoint linear operator on the Hilbert space of $L^{2} p$-forms, which puts severe restraints on its eigenvalues. In particular, $\operatorname{spect}\left(-\Delta_{p}\right) \subseteq[0 \infty)$. By the above theorem we see that the values of $s$ are constrained to lie in $[0, n] \cup([n-1] / 2-i \infty,[n-1] / 2+i \infty)$.

Definition. We shall denote by $S_{p}$ those values of $s \in \mathbf{C}$ corresponding to eigenvalues of the Laplacian $\Delta_{p}$ by the above theorem.

Example 1. For $p=0, \lambda=0, \Delta_{0}$ has a single (constant) eigenfunction, which corresponds to a positive measure (the Patterson-Sullivan measure [15]) on $S^{n-1}$.

Example 2. For $0 \leq p<n, \lambda=0$, the Laplacian $\Delta_{p}$ has eigenforms which span the real homology group $H^{p}(V, R)$ by the Hodge theorem [17]. These correspond to a $\beta_{p}$-dimensional space of currents $v$ on $S^{n-1}$ which transform by $g^{*} v=\|D g\|^{[n-1]} v$ (where, as usual, $\beta_{p}$ denotes the $p$ th betti number, i.e., the dimension of $H^{p}(V, R)$.)

The spectra of the Laplacians exhibit the following useful duality:

Lemma ("Poincaré duality").

$$
\operatorname{spect}\left(\Delta_{p}\right)=\operatorname{spect}\left(\Delta_{n-p}\right)
$$

The proof is essentially the same as the simple proof of Poincaré duality (i.e., $\Delta_{p}^{*}={ }^{*} \Delta_{n-p}$, c.f. $[17$, Chapter 6]).

\section{TRANSFER OPERATORS}

Now consider the action which $\Gamma$ induces on $S^{n-1}$. Using ideas from symbolic dynamics we can construct systems of the following kind.

Proposition 2. There exists a finite set of pairs $\left(U_{1}, g_{1}\right), \ldots,\left(U_{k}, g_{k}\right)$ with $U_{i} \subseteq S^{n-1}$ open, but with $\operatorname{int}\left(\partial U_{i}\right)=\varnothing$, and $g_{i} \in \Gamma(1 \leq i \leq k)$ such that :

(a) $s^{n-1}=\bigcup_{i} u_{i}$;

(b) $\left\|D_{x} g\right\| \geq \beta>1$ for all $x \in U_{i}$;

(c) if $g_{i} U_{i} \cap U_{j} \neq \varnothing$ then $g_{i} U_{i} \supseteq U_{j}$. 
(One useful approach to constructing such a cover was explained in detail in [13]. One first constructs Markov sections for the geodesic flow associated to $V$, in the sense of Bowen [1], say, and the $\left\{U_{i}\right\}$ correspond to projections of the "unstable parts" to infinity. This is similar to a construction explained to the author by Caroline Series).

Let $B_{p, k}$ denote the Banach space of $C^{k} p$-forms on $\mathscr{U}=\amalg_{i} U_{i}$ whose derivatives are uniformly bounded. Let $B_{p}$ be the Banach space of uniformly bounded real analytic $p$-forms on $\mathscr{U}$. Let $f: \mathscr{U} \rightarrow \mathscr{U}$ be the (real analytic) map defined by $f \mid U_{i}=g_{i}$.

For $s \in \mathbf{C}$ one can define a bounded linear operator $L_{s, p}$ on either $B_{p, k}$ or $B_{p}$, which we shall call the transfer operator, by

$$
L_{s, p} w(x)=\sum_{y=f^{-1} x}\left\|D_{y} f\right\|^{-s}\left[\left(f^{-1}\right)^{*} w\right](x), \quad \text { for } w \in B_{p, k} \text { or } B_{p} .
$$

(This operator was originally considered by Ruelle for analytic forms [14] and later by Tangermann for $C^{k}$-forms [16].)

Remark. Since $f$ is conformal we could write

$$
\left[\left(f^{-1}\right)^{*} w\right](x)=\left(\begin{array}{l}
n \\
p
\end{array}\right)\|D f\|^{p} w\left(f^{-1} x\right) .
$$

The foilowing is a (slight modification) of results of Ruelle [14] and Tangermann [16]).

Proposition 2. Let $s=\sigma+i$ and let $P(\sigma)=P(-\sigma \log \|D f\|)$, where $P$ denotes the topological pressure. Then:

(i) The spectral radius of $L_{s, p}$ is less than $P(\sigma) / \beta^{p}$.

(ii) The operator $L_{s, p}$ on $B_{p}$ is nuclear. The spectrum of $L_{s, p}$ on $B_{p, k}$ contains only isolated eigenvalues of finite multiplicity in the region $|z|>$ $P(\sigma) / \beta^{p k}$.

Nuclear operators are the analogue for Banach spaces of trace class operators for Hilbert spaces, c.f. [14]. In particular, they are compact operators and their spectra away from zero consist only of isolated eigenvalues of finite multiplicity. The spectra will coincide on the region $|z|>P(\sigma) / \beta^{k}$.

We refer to [14] for a discussion of the pressure function and observe the following facts:

(a) $\sigma \rightarrow P(\sigma)$ is strictly convex and monotonically decreasing;

(b) $P(n-1)=0$;

(c) $\left|P^{\prime}(n-1)\right| \geq \log \beta$.

See Figure 1.

Definition. Denote by $T_{p}$ those values of $s \in \mathbf{C}$ for which $L_{s, p}$ has unity as an eigenvalue (for all sufficiently large $k$, in the case of $B_{p, k}$ ).

Now we want to consider the relationship between $S_{p}$ and $T_{p}$. 


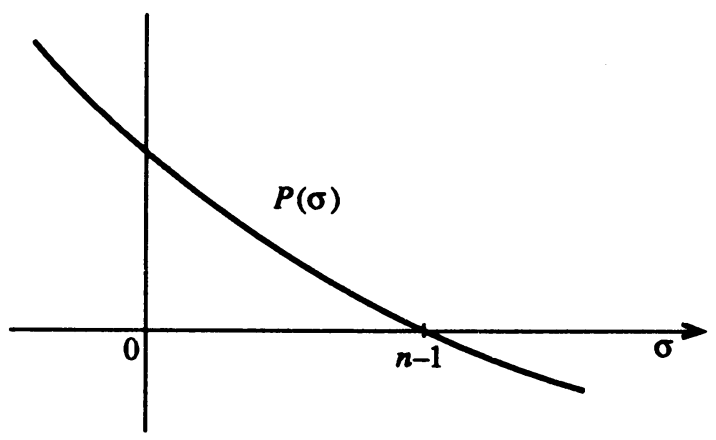

Figure 1

Theorem 2. $T_{p} \supseteq S_{p}$.

Proof. Assume that $s \in S_{p}$. By Theorem 1 one can deduce that there exists a current on $S^{n-1}$ such that $g^{*} v=\|D g\|^{s} v$, for all $g \in \Gamma$.

This current $v$ induces a current $v^{\prime}$ on each $U_{i} \subseteq S^{n-1}$ by the trivial inclusion, and thus induces a current on $\mathscr{U}$.

By evaluating the expression $L_{s, p}^{*} v^{\prime}$ on forms of sufficiently small support it reduces to $\|D f\|^{-s} f^{*} v^{\prime}$ on $U_{i}$. Furthermore, on $U_{i}, D f \mid U_{i}=D g_{i}$. By definition we know that $v$ satisfies $g_{i}^{*} v=\left\|D g_{i}\right\|^{s} v$ (whether we restrict to $U_{i}$ or not). Thus, by using a partition of unity (providing we take the opportunity to work in $B_{p, k}$ rather than $B_{p}$ ) and the above observations, we see that $L_{s, k} v^{\prime}=$ $v^{\prime}$. Therefore $s \in T_{p}$.

The obvious question is whether the inequality above is actually an equality. For the special case $n=2(p=0)$ this is true, because of the more precise form of Proposition 2 at our disposal. However, in the case $n>2$ this is far less likely, as we shall indicate in the next section.

\section{Zeta funCtions}

We can associate to each element $g \in \Gamma$ a norm $N(g)$ in one of two equivalent ways: (i) $N(g)=\left\|D_{x} g\right\|$, where $x$ is the unique fixed point for $g$ with $\left\|D_{x} g\right\|>1$; or (ii) $N(g)=e^{l}$, where $l$ is the length of the unique closed geodesic associated to the free homotopy class in $\pi_{1}(V)$ associated to $g \in \Gamma \cong \pi_{1}(V)$.

For a complex variable $s \in \mathbf{C}$ one can define a function, called a zeta function, by $\zeta(s)=\prod_{g \in \Gamma}\left(1-N(g)^{-s}\right)^{-1}$. (This converges for $\operatorname{Re}(s)>n-1$.)

For each $0 \leq p \leq n$, formally define a complex function for $f$ by

$$
\zeta_{p}(s)=\prod_{i}\left(1-\lambda_{i}(s)\right)^{-1}, \quad \text { where }\left\{\lambda_{i}(s)\right\}_{i} \text { are the eigenvalues of } L_{s, p} \text {. }
$$


If we consider the operators acting on real analytic forms then these operators are nuclear, and these zeta functions $\zeta_{p}(s)$ are well-defined and take the form

$$
\zeta(s)=\exp \sum_{m} \frac{1}{m} \operatorname{trace}\left[\left(L_{s, p} \mid B_{p}\right)^{m}\right] \quad \text { c.f. [14]. }
$$

Remark. It is worth observing that since $f$ is conformal we could write

$$
\operatorname{trace}\left[L_{s, p}^{m}\right]=\sum_{f^{m} x=x}\left(\begin{array}{l}
n \\
p
\end{array}\right)\left\|D_{x} f^{m}\right\|^{-[s-p]} / \operatorname{det}\left(1-D_{x} f^{m}\right) .
$$

Finally, combine the zeta functions $\zeta_{p}(s)$ to get a function

$$
\zeta(s)=\prod_{p} \zeta_{p}(s)^{(-1)^{p}} .
$$

This function can be expressed in the following terms:

\section{Lemma.}

$$
\zeta(s)=\exp \sum_{m} 1 / m \sum_{f^{m} x=x}\left\|D_{x} f^{m}\right\|^{S} \quad \text { (c.f. [14]). }
$$

Because of the above observation about conformality, this is the trivial "binomial" expansion $\left(1-\left\|D_{x} f^{m}\right\|\right)^{n}=\operatorname{det}\left(1-D_{x} f^{m}\right)$. (This is a particularly simple case of what is often called the "Atiyah-Bott" fixed point theorem for more general maps.)

We next want to relate the complex functions $\zeta(s)$ and $\zeta(s)$. This involves a result due to Bowen [2]:

Proposition 4. We can write $\zeta(s)=\zeta(s) N(s)$, where $N(s)$ is meromorphic in $\mathrm{C}$ and non-zero and analytic on $\operatorname{Re}(s)>n-1-\varepsilon$, for some $\varepsilon>0$. (In fact, $N(s)$ is a finite product of zeta functions similar to $\zeta(s))$.

The following result follows from either [14] or [17].

Proposition 5. For each $0 \leq p \leq n, \zeta_{p}(s)$ has an extension to $\mathbf{C}$ as an entire function. Furthermore, $s$ is a zero for $\zeta_{p}(s)$ if and only if $s \in T_{p}$.

We therefore see that $\zeta(s)$ has a meromorphic extension to $\mathbf{C}$. This is in fact well known by the classical approach using the Selberg trace formula. Fried showed the same result using the same ideas as above, except that he worked in the framework of the associated geodesic flow [6]. The viewpoint above appears more satisfactory for commenting on the poles of $\zeta(s)$ in light of the previous sections.

By Proposition 4 and the estimate in Proposition 2 on the spectral radius of transfer operators, we see that there exists $\varepsilon>0$ such that $\zeta_{p}(s)$ is non-zero and analytic for $\operatorname{Re}(s)>n-1-\varepsilon$. Thus the poles for $\zeta(s)$ in this region come 
only from $\zeta_{0}(s)$. However, more generally on $\mathbf{C}$ there will be contributions from the other zeta functions $\zeta_{p}(s)$ and from $N(s)$.

Using the Hadamard factorisation for the entire functions (of order one c.f. [14]), we can write:

$$
\zeta_{p}(s)=\prod_{\xi \in T_{p}}(1-s / \xi) e^{s / \xi} e^{A(p)}, \quad \text { where } A(p) \text { are constants }
$$

Thus we can write $\zeta(s)=\prod_{p} \prod_{\xi \in T_{p}}\left[(1-s / \zeta) e^{s / \xi} e^{A(p)}\right]^{(-1)^{p}}$. In view of Theorem 1 and "Poincare duality", there is an involution on $S_{p}$ given by $*: S_{p} \rightarrow S_{n-p}$ $\left(=S_{p}\right)$, such that $* \xi=n-\xi$. The logarithmic derivative of $\zeta(s)$ has a dependence on $s$ through a sum over terms $(-1)^{p} /(\xi-s), \xi \in T_{p}$, and a contribution from $N^{\prime}(s) / N(s)$. If $\xi \in S_{p} \subseteq T_{p}$, then we observe that, under the transformation $s \rightarrow n-s, \xi \rightarrow \xi^{*},(-1)^{p} /(s-\xi) \rightarrow(-1)^{p+1} /\left(s-\xi^{*}\right)=$ $(-1)^{n-1}(-1)^{n-p} /\left(s-\xi^{*}\right)$. Therefore:

Proposition 6. We always have one of the following identities:

$$
\begin{aligned}
\zeta^{\prime} / \zeta(s) & \pm \zeta^{\prime} / \zeta(n-s) \\
= & \sum_{p}(-1)^{p}\left(\sum_{\xi \in \in_{T-s_{p}}} 1 /(s-\xi)+\sum_{\xi \in T_{p}} 1 / \xi+A(p)\right) \\
& +N^{\prime} / N(s) \pm N^{\prime} / N(n-s),
\end{aligned}
$$

where we take the sum of the logarithmic derivatives if $n$ is even and the difference of the logarithmic derivatives if $n$ is odd.

Gangolli gives a functional equation for $\left(\zeta^{\prime} / \zeta\right)(s)$ involving the left hand side of the expression in Proposition 6 [8]. In particular, we expect exact cancellation to occur for the poles contributed by the terms on the right hand side of this equality (except for the "trivial" poles). From the study of symbolic dynamics we expect the contribution from $N^{\prime} / N$ to be non-trivial and thus that $S_{p} \subseteq T_{p}$ is a strict inclusion [2] (except in the very special case $n=2$ ).

\section{SCHOTTKY GROUPS}

Now consider the case where the manifold is no longer compact. More specifically, assume that $\Gamma$ has generators and inverses $g_{1}^{ \pm 1}, \ldots, g_{k}^{ \pm 1}$, such that the regions $U_{i}^{ \pm}=\left\{x \in S^{n-1} \mid\left\|D_{x} g_{i}^{ \pm 1}\right\|>1\right\}$ are piecewise disjoint. If the closures of these regions are also disjoint, then the map $f: \mathscr{U} \rightarrow S^{n-1}$, where $\mathscr{U}=\bigcap_{i} U_{i}^{ \pm}$and defined by $f \mid U_{i}=g_{i}$, has derivative bounded away from unity by $\beta>1$, say, if we shrink the regions $U_{i}^{ \pm}$slightly. 
The analogue of Theorem 1 is:

Proposition 7. There is a bijection between :

(a) co-closed eigenforms $\Delta_{p} w=-\lambda w$; and

(b) analytic currents on $S^{n-1}$ which transform as

$$
g^{*} v=\|D g\|^{s} v, \quad \text { where } s=[n-1] / 2+i \beta, \lambda=\left([n-1]^{2} / 4+\beta^{2}\right) \text {. }
$$

Let $S_{p}$ denote those $s \in \mathbf{C}$ which occur in Proposition 7 .

Under these conditions we shall let $B_{p}$ denote the Banach space of $p$-forms on $\mathscr{U}$ whose coefficients are analytic and uniformly bounded. As before we can define a transfer operator $L_{s, p}: B_{p} \rightarrow B_{p}, s \in \mathbf{C}, 0 \leq p \leq n$.

The following result is similar to part of Proposition 2 .

Proposition 8. The operators $L_{s, p}: B_{p} \rightarrow B_{p}$ are nuclear.

Let $L_{s, p}^{*}: B_{p}^{*} \rightarrow B_{p}^{*}$ denote the dual operator.

As before define $T_{p}$ to be the set of $s \in \mathbf{C}$ such that unity is an eigenvalue of $L_{s, p}$ (and thus by duality of $L_{s, p}^{*}$ ). The first part of Proposition 2 carries over to this setting:

Proposition 9. Let $s=\sigma+$ it and let $P(\sigma)=P(-\sigma \log \|D f\|)$; then the spectral radius of $L_{s, p}$ is bounded above by $P(\sigma) / \beta^{p}$.

We observe that:

(a) $\sigma \rightarrow P(\sigma)$ is strictly convex and monotonically decreasing;

(b) $P(\delta)=0$, where $\delta$ is the Hausdorff dimension of the limit set $\Lambda$;

(c) $\left|P^{\prime}(\delta)\right| \geq \log \beta$.

\section{Proposition 10. $S_{p} \supseteq T_{p}$.}

The proof of this inclusion is immediate from the inclusion of the domains. (The reason we cannot expect an equality in general is that $\Delta_{p}$ will have continuous spectra, and thus, in particular, $S_{p}$ will not even be countable.)

We can repeat the definitions of the complex functions $\zeta(s)$ and $\zeta_{p}(s)$ as in the previous sections. In the present context we immediately write $\zeta(s)=$ $\prod \zeta_{p}(s)^{(-1)^{p}}$, there being no need for an analogue of Proposition 4 since here the symbolic dynamics are particularly elementary.

The following result follows from the techniques in [14].

Theorem 3. $\zeta(s)$ has a meromorphic extension to $\mathbf{C}$.

Remark. The trace formula approach to $\zeta(s)$ does not seem particularly successful in proving results of the above form. The reason is that, for $V$ a manifold of infinite volume, the Laplacian has continuous spectrum [9]. By contrast, this thermodynamic approach becomes even easier.

\section{APPENDiX. Estimation of eigenVAlues}

In this appendix we shall propose some different approaches to estimating eigenvalues of the Laplacian. 
(a) The characterisation in terms of transfer operators would appear particularly useful. These can be approximated by finite rank operators and analysed numerically. For Schottky groups this should characterise small eigenvalues of the Laplacian (with co-closed eigenforms.) For the case where $V$ was compact this approach may result in additional values because of the inclusion $S_{p} \supseteq T_{p}$.

This approach to eigenvalues has some interesting consequences. Consider a (smooth) family $\Gamma_{\alpha}$ of Schottky groups (it is rather pointless considering groups with $V$ compact because of Mostow rigidity.) We shall be interested in the dependence of the values in $T_{p}$ on the parameter $\alpha$. In particular, this will include the Hausdorff dimension $\delta$ of the limit set as a special case. For $s \in T_{\alpha}$, a simple value, one can compute the derivative:

$$
d s / d \alpha=\frac{v\left[d\left(\log \left\|D f_{\alpha}\right\|\right) / d \alpha\right]}{v\left[\log \left\|D_{f \alpha}\right\|\right]}
$$

where $v$ denotes the eigencurrent $L_{s, p}^{*} v=v$. (This simply uses the implicit function theorem). For the case of the Hausdorff dimension $\delta$ of $\Gamma, v$ is the Patterson-Sullivan measure.

(b) A technique frequently used in estimating small eigenvalues of the Laplacian is to "approximate" the manifold by a graph and then solve the simpler problem in that context, and finally relate the solution back to the manifold. (One such typical approximation technique involves reducing the manifold into "thick" and "thin" components). Motivated by this we consider, instead of the Laplacian, the integral operator $U$ (on $L^{2}$ functions, say) with respect to the heat kernal $K_{t}(x, y)$, i.e., the probability density of a Brownian path starting at $x$ reaching $y$ at time $t$. This operator has eigenvalues $e^{-|\lambda| n}$ for compact manifolds. If we take a suitably fine partition $\left\{\alpha_{1}, \ldots, \alpha_{n}\right\}$ of the manifold for some fixed $t$, then $K_{t}(x, y)$ will be approximately constant for $x \in \alpha_{i}, y \in \alpha_{j}$. Thus $U$ can be approximated by an $n \times n$ matrix. By perturbation theory the large eigenvalues of $U$ corresponding to the small eigenvalues of $\Delta_{p}$ will be approximated by the eigenvalues of the matrix. The eigenvalues of this matrix can then, in principle, be numerically computed.

(c) Probably the most well-known approach to computing lower bounds on eigenvalues (for $p=0$ ) is the Cheeger isoperimetric constant [4].

We shall now consider a concrete problem due to Phillips and Sarnak [12] and propose an approach using this technique.

Assume that we take the case $n=3$ and let $\Gamma$ be a Schottky group generated by reflections in hyperplanes.

To begin, assume that the hyperplanes meet the sphere $S^{2}$ in a family of $n$ circles such that the region exterior to the circles in $S^{2}$ consists only of a triangle with circular arcs as sides, c.f. [2]. We can triangulate the sphere by triangles whose vertices are precisely the centres of our family of $n$ circles. For each such triangle $T$, corresponding to circles $C_{1}, C_{2}, C_{3}$, say, we can "inscribe" a 


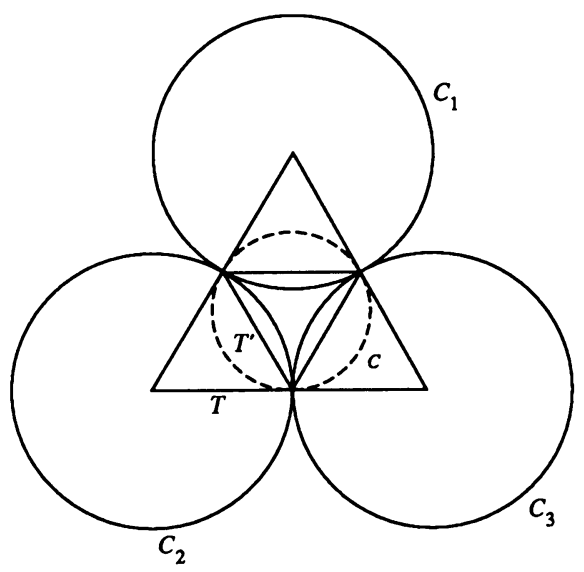

FIGURE 2

second smaller triangle $T^{\prime}$ whose vertices are the intersections of $T$ with the original circles. See Figure 2.

Finally, we can introduce a circle $C$ contained in $T$, but containing $T^{\prime}$, which passes through the vertices of $T^{\prime}$.

To bound below the first eigenvalue $\lambda$ of $\Delta_{0}$ for $V=H^{n} / \Gamma$, it suffices to do this for each of the pieces of an appropriate partition of $V$. (This is the Rayleigh method, where new boundaries should be given Neumann boundary conditions [4].) Fix the point $x_{0}$ of intersection between two circles in the original family and consider the decomposition of $V$ into regions bounded by the sides of a tetrahedron, with vertices $x_{0}$, and the three vertices of each triangle $T$.

For each such region $V$ we want to apply some form of the Cheeger inequality [4] (in the style of Brooks [3]) which should look like

$$
\lambda \geq \inf \left\{\operatorname{Area}(S) / \operatorname{Vol}\left(V_{\text {infinite }}\right)\right\},
$$

where $S$ runs through all finite area surfaces which divide $V$ into a finite value part, $V_{\text {finite }}$, and an infinite part, $V_{\text {infinite }}$.

By methods of geometric measure theory we expect that this infimum is realised by a surface $S$ (of constant mean curvature [4]). A natural candidate for $S$ is the triangle on the hemisphere above $C$ which projects down to the triangle $T^{\prime}$.

The area of $S$ can be calculated to be $2 \pi$ (independent of the geometry).

The region $V_{\text {infinite }}$ is the union of a tetrahedron (with one face $S$ and vertex $x_{0}$ ) and three other regions. The total volume is less than twice the total maximum volume $\mathscr{V}$ of a hyperbolic tetrahedron (numerically $\mathscr{V}=$ $1.0149416 \ldots)$.

This was all subject to the assumption that the circles lay in a certain configuration. However, Brooks has shown that any configuration, after an arbitrarily 
small perturbation, can take this form after adding additional circles [2]. Thus one expects this bound to hold in all cases by some version of monotonicity and continuity of the eigenvalues.

The above argument is largely heuristic, and so we make our statement as a conjecture:

Conjecture. $\lambda \geq \pi / 8 \mathscr{V}=.3869179 \ldots$. Phillips and Sarnak conjectured that $\lambda$ was bounded below independently of $\Gamma$ (which was subsequently proved in [5]) and gave numerical evidence that suggested that this lower bound was less than $7 / 16=.4375$.

\section{REFERENCES}

1. R. Bowen, Symbolic dynamics for hyperbolic flows, Amer. J. Math. 95 (1973), 429-460.

2. R. Brooks, On the deformation theory of classical Schottky groups, Duke J. Math. 52 (1985), 1009-1024.

3. Injectivity radius and low eigenvalues of hyperbolic manifolds, J. Reine Angew. Math. 390 (1988), 117-129.

4. I. Cheval, Eigenvalues in Riemannian geometry, Academic Press, New York, 1984.

5. P. Doyle, The bass note of a Schottky group, Acta Math. 160 (1988), 249-284.

6. D. Fried, The zeta functions of Ruelle and Selberg I, Ann. Ec. Norm. Sup. 19 (1986), 491517.

7. P. Y. Gaillard, Transformation de Poisson de formes differentielles, Lé cas de l'espace hyperbolique, Comm. Math. Helvetici 61 (1986), 581-616.

8. R. Gangolli, Zeta functions of Selberg's type for compact space forms of symmetric spaces of rank one, Ill. J. Math. 21 (1977), 1-41.

9. L. Guillope, Sur la distribution des longueurs des geodesiques fermees $d^{\prime}$ une surface compacte a bord totalement geodesique, preprint.

10. S. Helgason, Topics in harmonic analysis on homogeneous spaces, Birkhäuser, Berlin, 1981.

11. S. Patterson, The limit set of a fuchsian group, Acta Math. 136 (1976), 241-273.

12. R. Phillips and P. Sarnak, The Laplacian for domains in hyperbolic space and limit sets of Kleinian groups, Acta Math. 155 (1985), 173-273.

13. M. Pollicott, Some applications of thermodynamic formalism to compact manifolds of constant negative curvature, Adv. in Math. (to appear).

14. D. Ruelle, Zeta functions for expanding maps and Anosov flows, Invent. Math. 34 (1976), 231-242.

15. D. Sullivan, The density at infinity of a compact group of hyperbolic motions, I.H.E.S. Publ. Math. 50 (1979), 171-202.

16. F. Tangermann, Meromorphic continuations of Ruelle zeta functions, Ph. D. thesis, Boston University, 1986.

17. F. Warner, Foundations of differentiable manifolds and Lie groups, Springer, Berlin, 1987.

Centro de Matematica, faculdade de Ciencias, Praca Gomes Teixeira, 4000 Porto, Portugal 